\title{
Relação entre criatividade e traços temperamentais em estudantes do ensino fundamental
}

\author{
Tatiana de Cássia Nakano - Pontifícia Universidade Católica - PUC-Campinas, Campinas, Brasil \\ Livia Rech de Castro - Pontifícia Universidade Católica - PUC-Campinas, Campinas, Brasil
}

\begin{abstract}
Resumo
A percepção da ausência de pesquisas nacionais que investiguem a relação entre criatividade e temperamento desenvolvidas com crianças e adolescentes motivou o presente estudo, conduzido junto a 142 estudantes de $6^{\circ}$ ao $9^{\circ}$ ano do Ensino Fundamental (84 do sexo feminino e 58 masculino), com idades entre 11 e 14 anos (M=12,8 anos; DP=1,10). Dois instrumentos foram utilizados: Teste de Criatividade Figural Infantil e Student Styles Questionnaire, cujos resultados indicaram que as dimensões temperamentais extroversão e intuição foram as que mais se relacionaram ao desempenho em criatividade (apresentando correlações significativas com os fatores Enriquecimento de Ideias, Preparação Criativa e Criatividade Total). Influência significativa da variável sexo foi encontrada em relação às dimensões racional e emotiva do temperamento, ao passo que a variável série e sua interação com sexo mostrou-se importante em um fator da criatividade (Aspectos Cognitivos).

Palavras-chave: Criatividade, Temperamento gênero, Série, Student Styles Questionnaire.
\end{abstract}

Relationship between creativity and temperamental traits in a sample of elementary school students

\begin{abstract}
The perception of lack of national research focused on investigating the relationship between creativity and temperament developed with children and adolescents motivated the present study, conducted among 142 students from $6^{\text {th }}$ to $9^{\text {th }}$ grade of elementary school (84 female and 58 male), aged between 11 and 14 years $(\mathrm{M}=12.8$ years; $\mathrm{SD}=1.10)$. Two instruments were used: Test of Figural Creativity for Children and Student Styles Questionnaire. The results indicated that the temperamental dimensions extraversion and intuition were the most related to performance on creativity (showing significant correlations with the factors Enrichment of Ideas, Creative Preparation and total creativity). Significant influence of gender was found in relation to rational and emotional dimensions of temperament, while the grade variable and its interaction with gender was found in Cognitive factor of creativity.
\end{abstract}

Keywords: Gender, School grade, Student Style Questionnaire.

Relación entre creatividad y rasgos temperamentales de estudiantes de educación básica

\begin{abstract}
Resumen
La percepción de la ausencia de investigaciones nacionales que estudien la relación entre creatividad y temperamento desarrollada con niños y adolescentes motivó el presente estudio. Participaron 142 estudiantes de sex to a noveno curso de la educación básica (84 mujeres y 58 hombres), con edades entre 11 y 14 años $(M=12,8$ años, $\mathrm{SD}=1,10)$. Se utilizaron dos instrumentos: Test de Creatividad Infantil Figurativo y el Student Styles Questionnaire, cuyos resultados indicaron que las dimensiones temperamentales extraversión e intuición fueron las que más se relacionaron al desempeño en creatividad (presentando correlaciones significativas con los factores (enriquecimiento de ideas, preparación creativa y el total de la creatividad). Influencia significativa de la variable sexo fue encontrada con relación a las dimensiones racional y emocional del temperamento, mientras que el curso y su interacción con el sexo se mostró importante en los aspectos cognitivos de la creatividad.

Palabras clave: Creatividad, Temperamento, Sexo, Curso, Student Styles Questionnaire.
\end{abstract}

Considerando-se a importância de se estudar as diferenças individuais, a literatura científica tem centrado suas investigações em três grandes domínios: intelecto (inteligência e criatividade), personalidade e temperamento (Digman \& Shmelyov, 1996). Pesquisadores têm tentado responder a uma importante questão: seriam esses três domínios independentes e, portanto, não relacionados, ou eles podem ser organizados em um modelo abrangente que inclui todos os três? Diante desse quadro, o presente trabalho foca-se na investigação da relação entre dois desses construtos, a saber, criatividade e temperamento, em uma amostra de crianças e adolescentes.
Diante da especificidade da faixa etária selecionada, a escolha do foco da pesquisa se dá em um contexto marcado pela existência de grande número de pesquisas nacionais e internacionais voltadas à investigação da relação entre criatividade e personalidade e de um número ainda escasso de estudos enfocando o primeiro construto e sua relação com temperamento. Isso porque, durante décadas de estudo da criatividade, destaque tem sido dado à investigação das características de personalidade presentes nas pessoas criativas, sendo este um dos aspectos que mais tem atraído a atenção dos pesquisadores da área (Stein, 1974), dada sua influência no funcionamento criativo (Novaes, 1972; Sternberg \& Lubart, 1996; Torrance \& Ball, 1990; Wallack \& 
Kogan, 1965; Wechsler, 2008). Características de personalidade associadas à realização criativa têm sido pesquisadas em vários domínios e em diferentes níveis etários, fazendo uso de uma variedade de procedimentos (Barron \& Harrington, 1981), visto que a capacidade de pensar de forma criativa e inovadora, aliada à apresentação de atributos de personalidade que se associam à criatividade, tem sido apontada como um importante recurso de enfrentamento dos desafios atuais (Alencar \& Fleith, 2008).

Esse tipo de avaliação tem sido direcionado em termos de quais seriam os atributos pessoais presentes nas pessoas eminentemente criativas, na tentativa de se compreender como se combinam características cognitivas, motivacionais e de personalidade nesses indivíduos (Alencar, 2007; Candeias, 2008). Desenvolvidos, em sua maior parte, de forma essencialmente exploratória (baseados em trabalhos biográficos sobre personalidades eminentes, indivíduos criativos descrevendo a si mesmos ou sendo descritos por seus pares), os estudos têm apontado ligações importantes entre a personalidade e a criatividade do indivíduo. Como resultado, o avanço das investigações que examinaram essa relação, desenvolvidas desde 1950, permitiu que alguns traços de personalidade que caracterizam pessoas criativas pudessem ser isolados (Lubart, 2007), de forma que a lista desses traços comumente encontrados em indivíduos criativos acabou por tornar-se mais exata, precisa e abrangente, conforme salientado por Haller e Courvoisier (2010).

A partir desses estudos, descrições da personalidade criativa passaram a ser apontadas na literatura e salientadas por autores como Guilford (1967), Romo (2008), Torrance (1966), Torrance e Ball (1990), Torrance e Safter (1999), Wallach e Kogan (1965) e Wechsler (2008). De acordo com esses autores, dentre as características descritoras da pessoa criativa, destacam-se, de forma bastante consensual, a importância da autoconfiança, abertura à experiência, capacidade de assumir riscos, tolerância à ambiguidade, independência de pensamentos e julgamentos, intuição, sentimento de destino criativo, dentre outras. Note-se que a semelhança nas descrições apontadas por diversos pesquisadores mostra que o trabalho empírico dos últimos 35 anos na temática aponta para um conjunto relativamente estável de características centrais, as quais continuam a emergir como correlatos de realização criativa em muitos domínios (Barron \& Harrington, 1981), de modo que seus resultados apontaram para a existência de uma consistente associação entre os dois construtos, embora diferenças possam ser encontradas (Feist, 1998).

Enquanto algumas teorias enfatizam a avaliação da criatividade centrada na pessoa, com referência à investigação de atributos pessoais, como inteligência e personalidade, outros pesquisadores ainda preferem considerá-la dentro de uma visão clínica, na qual a criatividade é considerada resultado de processos incomuns de personalidade, adaptados ou não (Batey \& Furnham, 2006). Dessa forma, o que se tem constatado, segundo Stumm, Chung e Furnham (2011), é a parcela de responsabilidade da personalidade nas variações substanciais na criatividade, dado o fato dos traços de personalidade apresentarem-se consistentemente relacionados aos componentes de competência criativa, principalmente aqueles relacionados aos aspectos de temperamento, características pessoais e atitudes emocionais (Gough, 1979).

Entretanto, embora essa temática já venha sendo, historicamente, bastante explorada, uma constatação importante refere-se ao fato de que, embora a literatura disponha de um grande número de estudos sobre a personalidade de adultos criativos, a personalidade de crianças altamente criativas quase não foi estudada, conforme salientado por De La Torre (2008), talvez pelo desafio que se constitui a avaliação de ambos os construtos nessa faixa etária. Especificamente em relação à personalidade, uma possível justificativa ampara-se na dificuldade de se avaliar esse construto nessa população. A solução encontrada pelos pesquisadores tem sido o estudo do temperamento, compreendido enquanto a base biológica das diferenças individuais presentes na reatividade emocional e fisiológica, que são expressas por meio de emotividade positiva ou negativa (Ganiban, Saudino, Ulbricht, Neiderhiser \& Reiss, 2008), ou seja, um padrão de comportamento que diferencia as pessoas entre si e as habilita a enfrentar as situações do cotidiano de forma característica e própria" (Guzzo, Primi \& Ito, 2003). Desse modo, constata-se que mais comumente tem se investigado o temperamento em amostras de crianças e adolescentes e a personalidade em adultos, visto que o primeiro construto pode influenciar e até mesmo restringir o desenvolvimento de outros aspectos do segundo (Digman \& Shmelyov, 1996).

Assim, o que se faz notar é que ambos os construtos encontram-se bastante relacionados na literatura, visto que os traços de temperamento têm sido definidos como "dimensões comportamentais e emocionais individuais que se desenvolvem na infância e coletivamente formam a base para a personalidade mais tarde" (Gagne, Hulle, Aksan, Essex \& Goldsmith, 2011, p. 337), mostrando-se estável bem precocemente na criança, entre os 24 e 48 meses (Lemery, Goldsmith, Klinnert \& Mrazek, 1999). Historica e teoricamente, tal construto vem sendo visualizado como um importante 
precursor do desenvolvimento de estrutura de personalidade, geralmente considerado o fundamento biológico e emocional em que a personalidade se baseia, ou seja, a primeira expressão das diferenças individuais na personalidade, segundo visão de Rothbart, Ahadi e Evans (2000). Ressalte-se, no entanto, que a forma como as diferenças de temperamento em crianças e jovens estão relacionadas com a personalidade mais tarde ainda não esteja clara. Uma possibilidade, segundo Graziano, JensenCampbell e Sullivan-Logan (1998), é a de que os processos biológicos que produzem a diferença de temperamento (por exemplo, os genes) também regulem tanto a expressão maturacional do comportamento quanto o temperamento na infância e posteriormente na personalidade.

Convém, entretanto, salientar que, embora a diferença entre as dimensões de temperamento e as de personalidade seja vaga, um estudo de metanálise conduzido por Roberts e DelVecchio (2000) mostrou a existência de relação entre temperamento e traços de personalidade em crianças, cuja magnitude mostrou-se influenciada pela idade do sujeito (sendo de 0,35 entre zero e 2,9 anos; 0,52 entre 3 e 5,9 anos; e de 0,45 entre 6 e 11,9 anos). A diferença entre os construtos, por outro lado, situaria-se no fato de que o temperamento mostra-se como o núcleo afetivo, activational, e de atenção da personalidade, enquanto esta inclui muito mais do que o temperamento, em particular $\mathrm{O}$ conteúdo do pensamento, habilidades, hábitos, valores, defesas, moral, crenças e cognições sociais (Janson \& Mathiesen, 2008).

Outra importante questão a ser salientada referese à uma tendência nos estudos sobre temperamento em focar principalmente os aspectos negativos apresentados por crianças problemáticas (Degnan \& colaboradores, 2011), ficando os aspectos positivos relegados a segundo plano. Diferentemente desse enfoque, o presente estudo almeja apresentar ambos os construtos dentro de uma visão positiva, compreendendo-os como aspectos saudáveis e potenciais do ser humano.

Isso porque o reconhecimento da existência de diferenças individuais na forma como estudantes mostram seu temperamento pode fornecer uma importante informação a ser utilizada no processo de identificação de talentos e no auxilio à superação de possíveis dificuldades (Oakland, Glutin \& Horton, 1996), bem como na compreensão do funcionamento adaptativo e não-adaptativo infantil (Berdan, Keane \& Calkins, 2008). Assim, seu estudo justifica-se perante a importância desse construto enquanto fator de proteção e fator de risco na infância, dada sua possível influência na manifestação de problemas comportamentais infantis (Janson \& Mathiesen, 2008), dificuldades sociais e acadêmicas (Curby, Rudasill, Edwards \& Pérez-Edgar, 2011; Hane, Fox, PolakToste, Ghera \& Guner, 2006), capacidade preditiva de ajustamento durante a passagem da infância para a adolescência (Lengua, 2006), prevenção da desadaptação durante a adolescência (Ganiban e colaboradores, 2008), sobre o humor e ansiedade infantil (Sellbom, Ben-Porath \& Bagby, 2008) e reações emocionais de medo (Braungart-Rieker, Hill-Soderlund \& Karrass, 2010).

Também o estudo da criatividade mostra-se importante, dada sua capacidade de promover a realização pessoal e profissional (Wechsler, 2008), sendo tal habilidade considerada um diferencial de pessoas que apresentam capacidade intelectual superior (Lins \& Miyata, 2008), um meio de promover consciência sobre potencialidades (Sakamoto, 2000), agente de mudança e rendimento escolar (Hayes, 2004) e facilitadora do bem-estar pessoal (De la Torre, 2005), de modo a justificar a importância pessoal e social dessa característica (Virgolim, 2007) e a necessidade de seu estímulo e valorização (Nakano \& Wechsler, 2006).

Desse modo, pode-se perceber que o conhecimento gerado com base na investigação desses construtos pode ser usado na promoção do desenvolvimento pessoal, educacional e social. Entretanto, indiferente aos benefícios que podem resultar desse tipo de investigação, o que se faz notar, conforme salientado anteriormente, é a ausência de estudos dessa natureza desenvolvidos junto a crianças e adolescentes, de forma que a maior parte das pesquisas vem sendo conduzidas visando à investigação da relação entre criatividade e personalidade em adultos. Tal quadro se faz presente tanto no contexto internacional (Batey, Chamorro-Premuzic \& Furnham, 2009; Batey \& Furnham, 2006; Batey, Furnham \& Saffiulina, 2010; Cheng, Kim \& Hull, 2010; Feist, 1998; Ivcevic \& Mayer, 2007; Silvia, Martin \& Nusbaum, 2009; Stumm, Chung \& Furnham, 2011) quanto no nacional (Pereira, 2006), cujos resultados têm apontado uma maior associação de alguns aspectos de personalidade à criatividade, podendo-se destacar, dentre esses, abertura à experiência e intuição.

Importante estudo de metanálise baseado nos resultados de 111 pesquisas sobre criatividade, foi conduzido por Ma (2009) e apontou um efeito de tamanho médio $(0,71)$ da abertura desse fator sobre a criatividade. Uma única pesquisa encontrada desenvolvida com crianças (estudantes do ensino fundamental) foi conduzida por Fleith e Alencar (2008), cujos resultados indicaram a existência de correlações positivas significativas entre criatividade e quatro dimensões do autoconceito (competência 
acadêmica, aceitação social, aparência física e conduta comportamental). Segundo as autoras, os resultados apontaram para a influência dos fatores ambientais e características individuais na promoção do potencial criativo.

Diante dos resultados das pesquisas que buscaram investigar a relação entre criatividade e personalidade e da lacuna existente em relação à investigação da relação entre criatividade $\mathrm{e}$ temperamento em crianças $\mathrm{e}$ adolescentes brasileiros, a presente pesquisa teve por objetivo identificar como as características de temperamento se relacionam com o desempenho criativo em uma amostra de estudantes do ensino fundamental.

\section{Participantes}

\section{Método}

A amostra foi constituída por 142 participantes, igualmente divididos entre os sexos, com idades entre 11 e 14 anos (média 12,8 anos e DP1,10), estudantes de sexto $(n=25)$, sétimo $(n=21)$, oitavo $(n=47)$ e nono $(n=49)$ ano do ensino fundamental, de duas escolas localizadas no estado de São Paulo, selecionadas por conveniência, sendo uma pública e uma particular.

\section{Instrumentos}

Teste de Criatividade Figural Infantil Nakano, Wechsler \& Primi, 2011)

Instrumento no qual os participantes são convidados a compor desenhos em três atividades a partir de estímulos pouco definidos. As características avaliadas pelo instrumento são 12: fluência, flexibilidade, elaboração, originalidade, expressão de emoção, fantasia, movimento, perspectiva incomum, perspectiva interna, uso de contexto, extensão de limites e títulos expressivos, agrupadas em quatro fatores, de acordo com a estrutura fatorial apontada no estudo de Nakano e Primi (2012): fator 1: Enriquecimento de Ideias, cujas características envolvem ver a situação de uma forma mais detalhada e o enriquecimento da resposta, visualizada em um contex to mais amplo e dinâmico; fator 2: Emotividade, composto por características que envolvem o uso de recursos criativos ligados a uma percepção mais emocional, facilitadora no processo de descoberta de uma nova ideia; fator 3: Preparação Criativa, considerado um fator de "aquecimento" dado o fato de contemplar somente características avaliadas na primeira atividade do instrumento, sendo, por esse motivo, visualizado como uma oportunidade de treino para a realização das atividades posteriores, que permitem o uso mais livre da criatividade e maior número de respostas; fator 4: Aspectos Cognitivos, composto por características criativas que fazem uso de recursos cognitivos que envolvem a busca de soluções diferenciadas, originais e que vão além dos limites estabelecidos, além de uma pontuação total no instrumento, resultante da soma da pontuação obtida nos quatro fatores.

Estudos visando à busca por evidências de validade e precisão do instrumento indicaram valores entre 0,81 e 0,94 de correlação para validade concorrente com o Teste Figural de Torrance e índices entre 0,84 e 0,95 de correlação para a precisão por meio do teste e reteste, ambos com intervalo de confiança de 95\% (Nakano \& Wechsler, 2006). Os quatro fatores apresentaram bom ajuste ao modelo, verificado por meio de estudos envolvendo uso da Teoria de Resposta ao Item (Nakano, Wechsler \& Primi, 2011), de maneira que os resultados das pesquisas apontam bons indicadores de precisão e validade em amostras brasileiras.

Student Styles Questionnaire (Oakland, Gluttin \& Horton, 1996)

A escala tem sua proposta de avaliação da personalidade por meio das dimensões bipolares de temperamento (introversão $\mathrm{x}$ extroversão, prático $\mathrm{x}$ imaginativo, pensamento $\mathrm{x}$ sentimento, organizado $\mathrm{x}$ flexível). O instrumento, voltado a crianças e jovens entre 8 e 17 anos, tem sido utilizado na investigação do temperamento de estudantes provenientes dos mais diferentes países (Oakland, Pretorius \& Lee, 2008).

Em sua versão original, as evidências de validade convergente foram confirmadas em estudo comparativo entre os resultados obtidos no SSQ e dois outros instrumentos que avaliam o mesmo construto (Myers-Briggs Type Indicador - MBTI e Murphy-Meisgeier Type Indicator for children - MMTIC), cujos resultados apontaram correlações significativas entre as dimensões avaliadas pelos instrumentos. A precisão foi avaliada por meio do teste e reteste, com coeficientes variando entre 0,67 e 0,80 (Oakland, Gluttin \& Horton, 1996). Análises fatoriais baseadas nos resultados de crianças de sete países mostraram uma estrutura fatorial estável, dando suporte ao uso internacional do instrumento (Benson, Oakland \& Shermis, 2009).

No Brasil, o instrumento foi aplicado por Riello (1992) em estudantes do ensino fundamental e médio. A análise fatorial por componentes principais e rotação varimax apontou a existência de oito fatores, distribuídos em dimensões dicotômicas de temperamento: Introversão x Extroversão, Crítica $\mathrm{x}$ Percepção, Intuição $\mathrm{x}$ Sensação e Sentimento $\mathrm{x}$ Pensamento, de forma a confirmar as dimensões originais do instrumento e evidenciar a validade conceitual destas subescalas em amostras brasileiras. Essa versão foi utilizada na presente pesquisa, sendo 
composta por 100 itens que apresentam duas alternativas de resposta cada, devendo o estudante escolher aquela que melhor o descreve.

\section{Procedimentos}

A presente pesquisa inicialmente foi submetida à avaliação do Comitê de Ética em Pesquisa, tendo sido aprovada sob número 0106.0.147.000-09. Após seleção das escolas por conveniência, a aplicação foi realizada em um único dia em cada escola, de forma coletiva em sala de aula. Os instrumentos foram corrigidos de forma a dar origem a pontuações brutas em cada um dos oito tipos de temperamento e nos quatro fatores da criatividade, os quais foram comparados, de forma a estimar a relação entre o desempenho em criatividade e temperamento. Análises também visaram estimar a influência das variáveis série e sexo nos dois instrumentos.

\section{Resultados e Discussão}

Inicialmente são apresentadas as estatísticas descritivas (médias e desvios padrão) por sexo e série, dispostas na Tabela 1, para as oito dimensões de temperamento e os quatro fatores da criatividade.

Tabela 1. Médias e desvios padrão por sexo e série escolar

\begin{tabular}{lcccccccccccc}
\hline \multicolumn{1}{c}{ Variável } & \multicolumn{3}{c}{ Fem. } & \multicolumn{1}{c}{ Masc. } & \multicolumn{2}{c}{$6^{\circ}$ ano } & \multicolumn{2}{c}{$7^{\circ}$ ano } & \multicolumn{2}{c}{$8^{\circ}$ ano } & \multicolumn{2}{c}{$9^{\circ}$ ano } \\
\hline Extroversão & 11,00 & 4,21 & 9,91 & 4,21 & 11,24 & 4,25 & 9,95 & 4,65 & 10,15 & 3,81 & 10,86 & 4,48 \\
Introversão & 9,00 & 4,21 & 9,93 & 4,15 & 8,72 & 4,24 & 9,95 & 4,56 & 9,72 & 3,75 & 9,14 & 4,48 \\
Sensação & 6,52 & 2,38 & 7,59 & 2,59 & 6,20 & 2,19 & 6,52 & 2,44 & 7,55 & 2,70 & 6,96 & 2,44 \\
Intuição & 8,44 & 2,35 & 7,36 & 2,66 & 8,80 & 2,19 & 8,43 & 2,33 & 7,38 & 2,74 & 8,00 & 2,47 \\
Percepção & 6,57 & 3,07 & 6,22 & 2,58 & 5,64 & 2,43 & 5,29 & 2,75 & 6,79 & 2,97 & 6,98 & 2,90 \\
Crítica & 7,42 & 3,08 & 7,72 & 2,54 & 8,36 & 2,43 & 8,67 & 2,83 & 7,19 & 2,96 & 6,98 & 2,85 \\
Pensamento & 6,92 & 1,87 & 8,24 & 1,88 & 7,48 & 1,93 & 8,05 & 1,49 & 7,43 & 1,93 & 7,22 & 2,22 \\
Sentimento & 5,06 & 1,88 & 3,71 & 1,85 & 4,48 & 1,89 & 3,90 & 1,48 & 4,53 & 1,94 & 4,76 & 2,23 \\
F1 & 47,06 & 22,13 & 42,64 & 17,79 & 47,84 & 19,21 & 40,52 & 13,86 & 43,38 & 24,56 & 47,76 & 19,27 \\
F2 & 2,85 & 4,38 & 2,17 & 2,98 & 2,84 & 3,69 & 2,00 & 2,02 & 2,85 & 4,59 & 2,41 & 3,87 \\
F3 & 5,69 & 4,85 & 5,07 & 4,31 & 3,96 & 2,77 & 3,67 & 3,07 & 5,13 & 4,34 & 7,24 & 5,59 \\
F4 & 45,17 & 14,80 & 43,33 & 16,48 & 53,12 & 19,31 & 43,10 & 15,72 & 46,34 & 14,77 & 38,69 & 11,33 \\
\hline Criat. total & 100,56 & 35,87 & 94,16 & 28,67 & 110,16 & 28,55 & 89,29 & 25,79 & 97,60 & 38,78 & 95,76 & 31,45 \\
\hline
\end{tabular}

De acordo com os dados apresentados, pode-se visualizar que, no instrumento de temperamento, o sexo feminino apresenta médias maiores que o masculino nas dimensões de extroversão, intuição, percepção e sentimento. Por outro lado, os homens se destacam em introversão, sensação, crítica e pensamento. Já em relação ao instrumento de criatividade, pode-se notar uma superioridade do sexo feminino nos quatro fatores (Enriquecimento de Id eias, Emotividade, Preparação Criativa e Aspectos Cognitivos), além da pontuação total.

Diante das aparentes diferenças de médias, a Análise Multivariada da Variância foi empregada e indicou que a variável sexo exerceu influência significativa nas dimensões pensamento $(\mathrm{F}=6,054$, $\mathrm{p} \leq 0,015)$ e na dimensão sentimento $(\mathrm{F}=6,232$, $\mathrm{p} \leq 0,014)$, confirmando a ideia disseminada no senso comum de que homens tendem a ser mais racionais e mulheres mais emotivas. Os resultados convergem para aqueles apresentados por Oakland, Joyce, Horton e Glutting (2000), nos quais tanto garotos superdotados como não-superdotados apresentaram preferência pelos estilos que envolvem pensamento, ao passo que as meninas indicaram preferência por estilos que envolvem mais sentimento. De acordo com Oakland e colaboradores (1996), essas diferenças começariam a aparecer por volta dos 8 anos, mantendo-se ao longo $\mathrm{da}$ idade adulta, podendo ainda ser universais, dado o fato de que, de uma forma geral, mais homens apresentam o tipo pensamento como temperamento, enquanto mais mulheres mostram-se sentimento. A mesma percepção é apresentada por León, Oakland, Wei e Berrios (2009).

Assim, considerando-se a descrição da dimensão pensamento, pode-se perceber que, nesse estudo, alunos do sexo masculino apresentaram maior tendência a se divertir analisando fatos e ideias, preferência por tomar decisões baseadas na lógica em detrimento do uso de sentimento, além de apresentarem gosto por competições, debates e preocupação com a justiça (Oakland, Alghorani \& Lee, 2007). Por causa dessas preferências, beneficiariam-se de estilos de ensino que envolvessem análise de dados por meio de contrastes e comparações, possíveis a 
partir do estímulo a jogos competitivos (Oakland e colaboradores, 2000).

Por outro lado, as alunas mostraram mais tendência a tomar decisões com base em valores pessoais subjetivos, dedicando-se a manter a harmonia, maior propensão a necessitar de elogio, ser simpática e diplomática, gosto por agradar as pessoas, considerando suas necessidades, assim como interesse pelas pessoas que estão por trás dos trabalhos e ideias (Oakland e colaboradores, 2007). Por essas características, se beneficiariam de métodos de ensino que envolvessem aprendizado cooperativo, reforço verbal e tutoria (Oakland e colaboradores, 2000).

Tais diferenças teriam origem, segundo Graziano e colaboradores (1998), nas diferentes expectativas sociais difundidas em relação ao comportamento de meninas e meninos. O mesmo traço de temperamento pode ser interpretado, de acordo com os autores, de maneiras diferentes, dependendo de quem o apresenta. Essas diferenças em temperamento em virtude do gênero também foram confirmadas por Else-Quest, Hyde, Goldsmith e Van Hulle (2006) em estudo de metanálise realizado a partir de 189 pesquisas, cujos resultados indicaram a existência de padrões distintos de temperamento entre meninos e meninas, principalmente em relação a dimensões relacionadas ao controle de esforço e afetividade negativa.

A influência da variável série escolar também mostrou-se significativa no fator 4 (aspectos cognitivos) da criatividade $(\mathrm{F}=3,126, \mathrm{p} \leq 0,028)$, assim como a interação entre sexo e série, nesse mesmo fator $(\mathrm{F}=2,889, \mathrm{p} \leq 0,038)$. Note-se que a influência da série ocorre somente em relação ao instrumento de criatividade, não se mostrando significativa em relação às medidas do instrumento de temperamento.

De acordo com a descrição desse fator, diferenças poderiam ser sentidas entre os gêneros e alunos de diferentes séries, principalmente em relação às características criativas que fazem uso de recursos cognitivos, que envolvem a busca de soluções diferenciadas, originais e que vão além dos limites estabelecidos. Essas diferenças poderiam ser sentidas na forma como os alunos lidam com problemas incompletos ou sem solução. Isso porque as pessoas que apresentam aspectos cognitivos criativos bem desenvolvidos tendem a considerar os fatores importantes envolvidos no problema antes de contentarem-se com a primeira solução como forma de buscar soluções melhores e produzir resultados mais satisfatórios, fazendo uso de recursos como redefinição de problemas, busca por soluções diversificadas e originais, de maneira a quebrar o jeito habitual de pensar. Por outro lado, pessoas com baixas habilidades geralmente podem ser descritas como aquelas que buscam soluções comuns, imaginando o que a maioria das pessoas faria para solucionar aquele problema. Tendem a buscar sempre as mesmas soluções, preferencialmente aquelas que já tenham funcionado em alguma outra ocasião (Nakano e colaboradores, 2011). Nesse sentido, como forma de desenvolver os aspectos cognitivos da criatividade, deve-se estimular a pessoa a pensar em soluções que ela imagina serem só suas, a procurar por opções diferentes daquelas que acha que as demais pessoas irão pensar, além de incentivo à busca de melhores soluções, a fim de produzir resultados mais satisfatórios que aqueles encontrados sob impulso.

Uma segunda análise, visando identificar a relação entre temperamento e criatividade, foi realizada fazendo-se uso da correlação de Pearson, calculada para cada uma das oito dimensões do temperamento e os quatro fatores da criatividade, além da sua pontuação total. Os resultados são apresentados na Tabela 2.

Tabela 2. Correlação de Pearson entre os fatores do teste de criatividade e as dimensões do temperamento (continua)

\begin{tabular}{ccccccc}
\hline Variável & & F1 & F2 & F3 & F4 & Criat. Total \\
\hline Extroversão & Correlação de Pearson & $0,199^{*}$ & 0,083 & $0,166^{*}$ & 0,070 & $0,185^{*}$ \\
& Significância & 0,018 & 0,329 & 0,049 & 0,405 & 0,027 \\
Introversão & Correlação de Pearson & $-0,189^{*}$ & $-0,076$ & $-0,160$ & $-0,076$ & $-0,180^{*}$ \\
& Significância & 0,024 & 0,368 & 0,057 & 0,366 & 0,032 \\
\multirow{2}{*}{ Sensação } & Correlação de Pearson & $-0,201^{*}$ & $-0,134$ & $-0,245^{* *}$ & $-0,022$ & $-0,195^{*}$ \\
& Significância & 0,017 & 0,113 & 0,003 & 0,799 & 0,020 \\
Intuição & Correlação de Pearson & $0,213^{*}$ & 0,126 & $0,249^{* *}$ & 0,035 & $0,209^{*}$ \\
& Significância & 0,011 & 0,136 & 0,003 & 0,675 & 0,012 \\
\hline
\end{tabular}


Tabela 2. Correlação de Pearson entre os fatores do teste de criatividade e as dimensões do temperamento (continuação)

\begin{tabular}{ccccccc}
\hline Variável & & F1 & F2 & F3 & F4 & Criat. Total \\
\hline Percepção & Correlação de Pearson & 0,094 & 0,049 & 0,019 & 0,131 & 0,124 \\
& Significância & 0,266 & 0,562 & 0,825 & 0,120 & 0,143 \\
Crítica & Correlação de Pearson & $-0,088$ & $-0,044$ & $-0,011$ & $-0,128$ & $-0,117$ \\
& Significância & 0,297 & 0,603 & 0,898 & 0,128 & 0,165 \\
\multirow{3}{*}{ Pensamento } & Correlação de Pearson & $-0,077$ & 0,034 & $-0,106$ & $-0,185^{*}$ & $-0,138$ \\
& Significância & 0,361 & 0,687 & 0,211 & 0,027 & 0,101 \\
\multirow{2}{*}{ Sentimento } & Correlação de Pearson & 0,084 & $-0,034$ & 0,116 & $0,176^{*}$ & 0,140 \\
& Significância & 0,318 & 0,686 & 0,172 & 0,036 & 0,097 \\
\hline
\end{tabular}

Os resultados demonstraram que as dimensões extroversão e intuição mostraram-se as mais relacionadas à criatividade, apresentando correlações significativas com o fator 1 (enriquecimento de ideias), fator 3 (preparação criativa) e criatividade total. Os dados confirmam os resultados de pesquisa similar desenvolvida por Stumm e colaboradores (2011), a qual indicou que a dimensão extroversão contribuiu significativamente para uma alta realização criativa dos estudantes avaliados, assim como alunos que demonstravam personalidade do tipo intuição ou percepção apresentaram potencial mais criativo do que estudantes que tinham sensação ou julgamento como tipo de personalidade.

A descrição da dimensão extroversão aponta para pessoas que tendem a ter um lado bastante voltado ao social, que buscam ativamente por contatos pessoais e interação, apresentando ainda uma grande variedade de interesses, preferência por desenvolver suas id eias por meio da discussão com outras pessoas e aprendizagem mais proveitosa quando a mesma é desenvolvida por meio da fala e de atividades grupais cooperativas (Oakland, 2011; Oakland e colaboradores, 1996). Considerando sua combinação com as características de criatividade presentes nos fatores 1 e 3, pode-se visualizar que essa necessidade de interação social pode explicar, em partes, a preocupação apresentada por essas pessoas em fazer com que suas ideias sejam aceitas pelas demais, mesmo que isso envolva grandes doses de empenho, persistência, esforço e dedicação, típicas do indivíduo criativo. Por esse motivo costumam ser bastante persistentes em suas ideias, concentrando grande esforço no sentido de aperfeiçoálas ou melhorá-las, possuindo ainda boa capacidade de planejamento e organização, dada sua capacidade bem desenvolvida de enriquecimento de ideias (Nakano e colaboradores, 2011). Embora os extrovertidos sejam considerados mais impulsivos, fato que os deixa mais propensos a oferecer grande número de respostas, conduzindo a um aumento da fluência (Batey e colaboradores, 2009), a combinação entre esse traço de personalidade e a criatividade aponta para um indivíduo que perante um problema analisa as possibilidades de respostas e tem a calma suficiente para escolher, dentre as possibilidades, a melhor solução. Quando se veem diante de um problema que só permite a tentativa de uma única solução, apresentam certo nível de controle, o qual acaba por permitir o enriquecimento da ideia, de maneira a tornála mais atrativa (confirmado pelo bom desempenho em preparação criativa).

O segundo perfil, resultante da combinação entre a dimensão intuição e as características de criatividade presentes nos fatores 1 e 3 , nos leva a uma convergência de características. Um bom desempenho nesses fatores aponta para pessoas que gostam de aprender novas habilidades, planejar e trabalhar imaginando possibilidades, persistência em suas ideias e concentração de grande esforço tendo em vista aperfeiçoá-las ou melhorá-las, assim como a busca pela compreensão e solução dos problemas dentro de um contexto (Nakano, 2012a). Essas características criativas mostram-se facilitadas pela presença de outras tipicamente apresentadas por pessoas com dimensão intuição predominante, as quais envolveriam gosto pelo aprendizado de novas habilidades, pelo trabalho que envolve imaginação de possibilidades, pela realização de tarefas excessivamente complexas e seu interesse por mudanças, variedade, adaptações e surpresas, com foco em como as coisas podem ser melhoradas, e consequente evitação de formas repetidas de fazer as coisas (Benson e colaboradores, 2009; Oakland, 2011; Oakland e colaboradores, 2007; Oakland e colaboradores, 2000, Oakland e colaboradores, 2008). Assim vemos que a característica de personalidade intuição exerce influência bastante positiva na expressão criativa dos sujeitos que apresentam esse perfil.

A análise também mostrou que a dimensão sentimento correlaciona-se significativamente com o 
desempenho no fator 4 da criatividade (aspectos cognitivos). Pessoas com alta habilidade cognitiva criativa apresentam como característica de personalidade a abertura psicológica, sendo que, nesse caso, abertura voltada às pessoas. Para esse tipo de indivíduo, o processo de tomada de decisões se dá considerando o efeito das escolhas sobre as pessoas, ao invés de julgamentos baseados na lógica ou racionalidade, como faria uma pessoa com a função pensamento mais desenvolvida (Oakland, 2011). Diante de problemas incompletos ou sem solução, a pessoa criativa com tipo sentimento, antes de buscar uma solução, tenderia a considerar os fatores importantes envolvidos no problema, por meio do uso de um lado mais sentimental, considerando, principalmente, as consequências para as pessoas envolvidas.

Como uma dimensão oposta à extroversão, a qual apontou correlação significativa com a criatividade, a introversão mostrou-se negativamente correlacionada com o fator 1 da criatividade (enriquecimento de ideias) e com a criatividade total. Assim, em contrapartida, estudantes que apresentam estilo introvertido geralmente costumam ter poucos amigos íntimos, preferindo passar a maior parte do tempo sozinhos. Apresentam ainda interesses bem desenvolvidos, podendo ser descritos como hesitantes no compartilhamento de suas ideias com outras pessoas, embora gostem de ser reconhecidos pelo seu trabalho cuidadoso, aprendendo melhor quando são dadas oportunidades para pensar sobre o que está sendo ensinado (Oakland, 2011). Entretanto, sua dificuldade em manter contatos com outras pessoas, cujos feedbacks poderiam contribuir para o processo de aperfeiçoamento e enriquecimento da ideia, acaba por levá-las a baixa capacidade de enriquecimento de ideias, visto que crianças introvertidas tendem a gastar muito tempo tomando decisões (Oakland e colaboradores, 1996), de forma a apresentar nível de elaboração incompleto ou insatisfatório, o qual pode acabar por esconder ou anular todo o esforço criativo.

Como a pessoa introvertida apresenta tendência a evitar contatos pessoais, pode-se pensar na hipótese de que provavelmente elas apresentem baixo desempenho em enriquecimento de ideias pelo fato de não mostrar preocupação em comunicá-las aos demais, agravado pela presença de comportamentos de hesitação relacionados ao compartilhamento das suas ideias, assim como dificuldade em considerar o ponto de vista de outras pessoas (Nakano e colaboradores, 2011). Por esse motivo podem acabar agindo de forma impulsiva, sem grande esforço ou tentativa de aperfeiçoar suas ideias. Também demonstram dificuldade em considerar o contexto da situação, de forma que apresentam tendência a ver os problemas isoladamente, comportamento compreensível diante da sua preferência por privacidade e trabalho individualizado (Nakano, 2012b). Provavelmente o fato de ser mais introvertido faz com que a pessoa tenha maior dificuldade em expressar um grande número de ideias pelo fato de ser mais contida e apresentar-se mais voltada ao seu mundo interno e à reflexão, de forma que essa característica acaba por prejudicá-la na capacidade de enriquecimento de ideias e na expressão criativa total. A dificuldade interpessoal apresentada por esses indivíduos acaba por prejudicar o reconhecimento da sua produção criativa, a qual se dá, necessariamente, por meio do reconhecimento social.

Correlação negativa também foi encontrada entre a dimensão sensação e os fatores 1 e 3 da criatividade, além de seu total. Esse resultado corrobora as conclusões de Cheng e colaboradores (2010), segundo os quais, os alunos que demonstravam personalidade do tipo sensação ou julgamento apresentaram potencial criativo mais baixo do que estudantes que tinham intuição ou percepção como tipo de temperamento. A combinação entre os dois perfis, criativo e temperamental, mostra que a busca por praticidade e simplificação de tarefas e a falta de confiança em suas inspirações (León e colaboradores, 2009; Okland, 2011) poderiam resultar na apresentação de suas ideias de forma incompleta, de acordo com a primeira solução que encontrasse para os problemas, o que os levaria a agir de forma impulsiva, sem fazer esforço ou tentar aperfeiçoar suas ideias. Pelo fato de focalizarem no presente aquilo em que trabalham, acabam por não planejar o tempo necessário, característica que, aliada ao fato de gostarem de maneiras estabelecidas de fazer as coisas, justifica a tendência que os indivíduos com esse tipo de temperamento apresentam de ver o problema sob um único ponto de vista, apresentando dificuldade em prestar atenção à sua dinâmica de funcionamento (Nakano, Wechsler \& Primi, 2011).

Da mesma forma, correlação negativa foi encontrada entre a dimensão pensamento e o fator 4 criativo (aspectos cognitivos). Essa característica temperamental indica uma tendência a antecipar ou predizer os efeitos lógicos das escolhas, a partir de princípios analíticos e racionais, comunicando-se de maneira breve e sequencial (Oakland e colaboradores, 1996). Isso explica a dificuldade de buscarem soluções originais, uma vez que agem de maneira a fazer uso das mesmas soluções, preferencialmente aquelas que já tenham funcionado em alguma outra ocasião. Uma vez que a solução tenha dado certo, ela será novamente utilizada, sempre que possível. Assim, embora tenha muitas ideias, em sua essência elas se propõem a resolver o problema sempre da mesma forma, sem que 
haja a testagem de outras possibilidades diferentes, em virtude, principalmente, do fato de estarem muito presos à sua tendência lógica, analítica e racional. Sua forma de comunicação, breve e sequencial, pode dar origem a ideias precoces, apresentadas, muitas vezes, de forma incompleta diante da necessidade de busca por uma solução imediata (Nakano, 2012a; Nakano e colaboradores, 2011).

Assim, os resultados encontrados na presente pesquisa apontam para as dimensões temperamentais extroversão e intuição como as que mais se relacionaram ao desempenho em criatividade (apresentando correlações significativas com os fatores 1,3 e criatividade total), sendo positiva também a relação da dimensão sentimento com a criatividade. Em um outro oposto, introversão e sensação apresentaram correlações negativas com os mesmos fatores, assim como a função pensamento, de forma que os resultados permitem afirmar a relação entre traços de temperamento e expressão criativa.

O reconhecimento dessas diferenças ampara-se na constatação de que, embora o professor não seja necessariamente capaz de determinar todas as variações nos estilos cognitivos de seus alunos, deveria estar ciente de que eles abordam problemas de diferentes maneiras. Enquanto alguns alunos podem necessitar de ajuda para aprender a reconhecer aspectos importantes e ignorar detalhes irrelevantes, outros podem parecer perdidos em situações menos estruturadas e necessitar de instruções claras, passo a passo, enquanto um terceiro grupo pode ser ótimo em organizar, mas menos sensível aos sentimentos dos outros e não tão efetivo em situações sociais (Woolfolk, 2000). Assim, dada a existência de várias maneiras de se expressar a criatividade (Wechsler, 1999), torna-se essencial o respeito ao estilo individual nos ambientes educacionais (Nakano, 2010). Do mesmo modo, o conhecimento do temperamento dos sujeitos se mostra promissor no sentido de permitir a compreensão de seu impacto sobre os comportamentos das crianças e adolescentes, assim como auxílio na orientação sobre seu desenvolvimento.

\section{Considerações finais}

A presente pesquisa teve como objetivo identificar como as características de temperamento se relacionam com o desempenho criativo em uma amostra de estudantes do Ensino Fundamental, visando, ainda, trazer uma contribuição para a discussão da temática, dada a constatação da existência de uma lacuna que se faz presente na literatura científica nacional. Os resultados indicaram a existência de algumas relações significativas entre os dois construtos, bem como a influência do gênero do participante na medida de temperamento e da série e sua interação com gênero na medida de criatividade. Ainda que tais resultados caminhem em direção ao que vem sendo encontrado na literatura internacional, recomenda-se cautela na interpretação dos dados pela limitação do estudo em relação à composição da amostra utilizada. Um pequeno número de participantes, com amplitude de idade bastante reduzida, provenientes de somente dois estabelecimentos de ensino, não pode ser tomado como representativo da população brasileira, de modo que outros estudos são incentivados.

Ainda assim, diversos são os benefícios que podem ser destacados e resultar da aplicação prática desse conhecimento, sendo importante ressaltar, por exemplo, sua utilização no contexto educacional. Neste, tanto o desenvolvimento de comportamentos criativos, como o estímulo dos alunos em direção à expressão de seus potenciais podem ser empregados visando a um impacto positivo no desempenho educacional e no desenvolvimento de interesses, de modo que os resultados da pesquisa levam à reflexão acerca da necessidade de se estimular a criatividade e respeitar as diferenças individuais no temperamento dos estudantes no contexto escolar. Torna-se importante, assim, destacar o papel que os professores exercem no desenvolvimento de seus alunos, a fim de que os mesmos possam estar preparados para identificar as preferências individuais de modo a estimular os alunos em direção às suas possibilidades máximas (Nakano, 2009; Nakano \& Wechsler, 2012; Wechsler, 2008).

A tomada de consciência dos professores acerca da importância da estimulação criativa e do reconhecimento da existência de uma diversidade de comportamentos em sala de aula, provenientes dos diferentes tipos de temperamento, poderá ser utilizada de forma a estimulá-los ao desenvolvimento de métodos de ensino mais diversificados, diferenciados, adequados, estimulantes e enriquecidos, de modo a quebrar a rotina escolar e ampliar o interesse dos alunos pelo aprendizado. Para o professor, a tomada de consciência sobre a importância da valorização da criatividade em sala de aula e da influência do temperamento na expressão criativa poderia ser utilizada no planejamento de intervenções que busquem maximizar os pontos fortes do aluno, atentando aos seus pontos fracos, sendo este também o objetivo da identificação da criatividade nos contextos escolares. Somente assim seus benefícios podem ser ampliados para todos os contextos em que o aluno encontra-se inserido, contribuindo para seu crescimento e realização pessoal. 
Dessa maneira, almeja-se que o conhecimento gerado a partir dos resultados das avaliações do temperamento e da criatividade dos estudantes possa auxiliar pais e professores a compreender a existência de diversificada gama de preferências individuais, de modo a permitir-lhes o desenvolvimento de ambientes que atendam aos diferentes estilos e interesses. Esperase, ainda, conforme enfatizado por Oakland e colaboradores (2007), que de posse dessas informações, estas possam ser utilizadas pelos adultos para compreender os caminhos que podem favorecer o sucesso das crianças ao longo da vida acadêmica, observar problemas que podem ocorrer caso elas sejam colocadas em ambientes incompatíveis com suas preferências e aconselhá-los sobre possíveis vocações viáveis e congruentes, a fim de ampliar suas possibilidades de obter satisfação pessoal e profissional.

Estudos futuros poderão explorar, de forma mais ampla, a estabilidade da estrutura do temperamento e da expressão criativa, de modo a explorar as mudanças e diferenciações que ocorrem, por exemplo, de acordo com a idade ou outras variáveis, tais como região de moradia ou tipo de escola. Os resultados provenientes da ampliação e diversificação da amostra e a utilização de diferentes instrumentos que avaliam esses construtos poderão ou não confirmar os dados aqui apresentados, de modo a contribuir para a discussão acerca da relação entre criatividade e temperamento em crianças e adolescentes brasileiros.

\section{Referências}

Alencar, E. M. L. S. (2007). Criatividade no contexto educacional: três décadas de pesquisa. Psicologia Teoria e Pesquisa, 23, n.spe, 45-49.

Alencar, E. M. L. S. \& Fleith, D. S. (2008). Barreiras à promoção da criatividade no ensino fundamental. Psicologia Teoria e Pesquisa, 24(1), 59-65.

Barron, F. \& Harrington, D. M. (1981). Creativity, intelligence and personality. Annual Review of Psychology, 32, 439-476.

Batey, M. \& Furnham, A. (2006). Creativity, intelligence, and personality: a critical review of the scattered literature. Genetic, Social and General Psychology Monographs, 132(4), 355-429.

Batey, M., Chamorro-Premuzic, T. \& Furnham, A. (2009). Intelligence and personality as predictors of divergente thinking: the role of general, fluid and crystallized intelligence. Thinking Skills and Creativity, 4, 60-69.

Batey, M., Furnham, A. \& Saffiullina, X. (2010). Intelligence, general knowledge and personality as predictors of creativity. Learning and Individual Differences, 20, 532-535.
Benson, N., Oakland, T. \& Shermis, N. (2009). Evidence for cross-national invariance of children's temperament structure. Journal of Psychoeducational Assessment, 27, 3-16.

Berdan, L. E., Keane, S. P. \& Calkins, S. D. (2008). Temperament and externalizing behavior: social preference and perceived acceptance as protective factors. Developmental Psychology, 44(4), 957-968.

Braungart-Rieker, J. M., Hill-Soderlund, A. L. \& Karrass, J. (2010). Fear and anger reactivity trajectories from 4 to 16 months: the roles of temperament, regulation, and maternal sensitivity. Developmental Psychology, 46(4), 791-804.

Candeias, A. A. (2008). Criatividade: perspectiva integrativa sobre o conceito e a sua avaliação. Em M.F. Morais \& S. Bahia (Orgs.), Criatividade: conceito, necessidades e intervenção (pp.41-64). Braga, Portugal: Psiquilibrios.

Cheng, Y., Kim, K. H. \& Hull, M. F. (2010). Comparisons of creative styles and personality types between american and taiwanese college students and the relationship between creative potential and personality types. Psychology of Aesthetics, creativity and the arts, 4(2), 103-112.

Curby, T. W., Rudasill, K. M., Edwards, T. \& PérezEdgar, K. (2011). The role of classroom quality in ameliorating the academic and social risks associated with difficult temperament. School Psychology Quarterly, 26(2), 175-188.

De la Torre, S. (2005). Dialogando com a criatividade. São Paulo: Madras.

De La Torre, S. (2008). Criatividade aplicada: recursos para uma formação criativa. São Paulo: Madras.

Degnan, K. A., Henderson, H. A., Moas, O. L., Hane, A. A. Reeb-Sutherland, B. C. \& Fox, N. A. (2011). Longitudinal stability of temperamental exuberance and social-emotional outcomes in early childhood. Developmental Psychology, 47(3), 765780 .

Digman, J. M. \& Shmelyov, A. G. (1996). The structure of temperament and personality in Russian children. Journal of Personality and Social Psychology, 71(2), 341-351.

Else-Quest, N. M., Hyde, J. S., Goldsmith, H. H. \& Van Hulle, C. A. (2006). Gender differences in temperament: a meta-analysis. Psychological Bulletin, 132(1), 33-72.

Feist, G. J. (1998). A meta-analysis of personality in scientific and artistic creativity. Personality and Social Psychology Review, (4), 290-309.

Fleith, D. S. \& Alencar, E. M. L. S. (2008). Características personológicas e fatores ambientais relacionados à criatividade do aluno do Ensino Fundamental. Avaliação Psicológica, 7(1), 35-44. 
Gagne, J. R., Hulle, C. A. V., Aksan, N., Essex, M. J. \& Goldsmith, H. H. (2011). Deriving childhood temperament measures from emotion-eliciting behavioral episodes: scale construction and initial validation. Psychological Assessment, 23(2), 337-353.

Ganiban, J. M., Saudino, K. J., Ulbricht, J., Neiderhiser, J. M. \& Reiss, D. (2008). Stability and change in temperament during adolescence. Journal of Personality and Social Psychology, 95(1), 222-236.

Graziano, W. G., Jensen-Campbell, L. A. \& SullivanLogan, G. M. (1998). Temperament, activity, and expectations for later personality development. Journal of Personality and Social Psychology, 74(5), 12661277.

Gough, H. G. (1979). A creative personality scale for the adjective checklist. Journal of Personality and Social Psychology, 37(8), 1398-1405.

Guilford, J. P. (1967). The nature of buman intelligence. Nova Iorque: McGraw-Hill.

Guzzo, R. S. L., Primi, R. \& Ito, P. C. P. (2003). PTS Pavlovian Temperament Survey, versao adolescente / adulto: consistência interna e normatização para a realidade brasileira. Estudos de Psicologia (Campinas), 8(3), 525-533.

Haller, C. S. \& Courvoisier, D. S. (2010). Personality and thinking style in different creative domains. Psychology of Aestetics, creativity and the arts, 4(3), 149160.

Hane, A. A., Fox, N. A., Polak-Toste, C., Ghera, M. M. \& Guner, B. M. (2006). Contextual Basis of Maternal Perceptions of Infant Temperament. Developmental Psychology, 42(6), 1077-1088.

Hayes, D. (2004). Understanding creativity and its implications for schools. Improving Schools, 7(3), 279-286.

Ivcevic, Z. \& Mayer, J. D. (2007). Creative types and personality. Imagination, cognition and personality, 26(1/2), 65-86.

Janson, H. \& Mathiesen, K. S. (2008). Temperament profiles from infancy to middle childhood: development and associations with behavior problems. Developmental Psychology, 44(5), 13141328.

Lemery, K. S., Goldsmith, H. H., Klinnert, M. D. \& Mrazek, D. A. (1999). Developmental models of infant and childhood temperament. Developmental Psychology, 35(1), 189-204.

Lengua, L. J. (2006). Growth in temperament and parenting as predictors of adjustment during children's transition to adolescence. Developmental Psychology, 42(5), 819-932.

León, C., Oakland, T., Wei, Y. \& Berrios, M. (2009). Venezuelan children temperament styles and comparison with their United States peers.
Interamerican Journal of Psychology, 43(1), 125-133.

Lins, J. S. C. \& Miyata, E. S. (2008). Avaliando a aprendizagem de criatividade em uma oficina pedagógica. Ensaio: Avaliação de Políticas Públicas Educacionais, 16(60), 455-468.

Lubart, T. (2007). Psicologia da criatividade. Porto Alegre: ArtMed.

Ma, H. (2009). The effect size of variables associated with creativity: a meta-analysis. Creativity Research Journal, 21(1), 30-42.

Nakano, T. C. (2009). Investigando a criatividade junto a professores: pesquisas brasileiras. Psicologia Escolar e Educacional, 13(1), 45-53.

Nakano, T. C. (2010). Estilos de pensar e criar em estudantes de psicologia: diferenças regionais?. Estudos e Pesquisas em Psicologia, 10(3), 682-699.

Nakano, T. C. (2012a). O desenho na expressão criativa: teste de criatividade figural infantil. Em S. M. Wechsler \& T. C. Nakano (Orgs.), O desenho infantil: forma de expressão cognitiva, criativa e emocional (pp.67-95). São Paulo: Casa do Psicólogo.

Nakano, T. C. (2012b). Teste de Criatividade Figural Infantil. Em C. S. Hutz (Org.), Avanços em Avaliação Psicológica e Neuropsicológica de Crianças e Adolescentes (pp.425-451). São Paulo: Casa do Psicólogo.

Nakano, T. C. \& Primi, R. (2012). Teste de criatividade figural infantil: estrutura fatorial. Psicologia Teoria e Pesquisa.

Nakano, T. C. \& Wechsler, S. M. (2006). Teste Brasileiro de Criatividade Figural: proposta de instrumento. Revista Interamericana de Psicologia, 40(1), 103-110.

Nakano, T. C. \& Wechsler, S. M. (2012). Criatividade: definições, modelos e formas de avaliação. Em C. S. Hutz (Org.). Avanços em avaliação psicológica e neuropsicológica de crianças e adolescentes (pp. 327-361). São Paulo: Casa do Psicólogo.

Nakano, T. C., Wechsler, S. M. \& Primi, R. (2011). Teste de criatividade figural infantil. São Paulo: Vetor.

Novaes, M. H. (1972). Psicologia da criatividade. Rio de Janeiro: Vozes.

Oakland, T., Alghorani, M. A. \& Lee, D. H. (2007). Temperament-based learning styles of Palestinian and US children. School Psychology International, 28, $110-128$.

Oakland, T. (2011). Considerando qualidade de temperamento no ensino de crianças e jovens talentosos. Em S.M. Wechsler \& V.L.T. Souza (Orgs.), Criatividade e aprendizagem: caminhos e descobertas em perspectiva internacional (pp. 149-174). São Paulo: Loyola. 
Oakland, T., Glutting, J. J. \& Horton, C. B. (1996). Student styles questionnaire: manual. San Antonio: The Psychological Corporation.

Oakland, T., Joyce, D., Horton, C. B. \& Glutting, J. (2000). Temperament-based learning styles of identified gifted and nongifted students. Gifted Child Quarterly, 44(3), 183-189.

Oakland, T., Pretorius, J. D. \& Lee, D. H. (2008). Temperament styles of children from south Africa and the United States. School Psychology International, 29, 627-639.

Pereira, D. F. (2006). Um estudo sobre o Wartegg como medida de criatividade em seleção de pessoal (Dissertação de Mestrado não publicada). Faculdade de Psicologia da Universidade Federal do Rio Grande do Sul, Porto Alegre, Rio Grande do Sul.

Riello, I. C.(1992) O temperamento de adolescentes: estudo de um instrumento de avaliação (Dissertação de Mestrado não publicada). Faculdade de Psicologia da Pontifícia Universidade Católica de Campinas, Campinas, São Paulo.

Roberts, B. W. \& DelVecchio, W. F. (2000). The rankorder consistency of personality from childhood to old age: a quantitative review of longitudinal studies. Psychological Bulletin, 126, 3-25.

Romo, M. (2008). Creatividad em los domínios artístico y cientifico y sus correlatos educativos. Em M.F. Morais \& S. Bahia (Orgs.), Criatividade: conceitos, necessidades e intervenção (pp. 65-90). Minho: Psiquilibrios.

Rothbart, M. K., Ahadi, S. A. \& Evans, D. E. (2000). Temperament and personality: origins and outcomes. Journal of Personality and Social Psychology, 78(1), 122-135.

Sakamoto, C. K. (2000). Criatividade: uma visão integradora. Psicologia: Teoria e Prática, 2(1), 50-58.

Sellbom, M., Ben-Porath, Y. S., Bagby, R. M. (2008). On the hierarchical structure of mood and anxiety disorders: confirmatory evidence and elaboration of a model of temperament markers. Journal of Abnormal Psychology, 117(3), 576-590.

Silvia, P. J., Martin, C. \& Nusbaum, E. C. (2009). A snapshot of creativity: evaluating a quick and simple method for assessing divergent thinking. Thinking Skills and Creativity, 4, 79-85.

Stein, M. I. (1974). Stimulating creativity. Nova Iorque: Academic Press.

Sternberg. R. J. \& Lubart, T. I. (1996). Investing in creativity. American Psychologist, 51, 677- 688.

Stumm, S.V., Chung, A. \& Furnham, A. (2011). Creative ability, creative ideation and latent classes of creative achievement: what is the role of personality?. Psychology of Aestetics, Creativity and the Arts, 5(1), 1-8.

Torrance, E. P. (1966). Torrance tests of creative thinking. Lexington: Personnel Press.

Torrance, E. P., Ball, O. E. (1990). Streamlined scoring and interpretation guide and norms manual verbal and figural form B. Bensenville: Scholastic Testing Service.

Torrance, E. P. \& Safter, H. T. (1999). Making the creative leap beyond. Buffalo, NY: Creative Education Foundation.

Virgolim, A. M. R. (2007). Talento criativo: expressão em múltiplos contextos. Brasilia: UNB.

Wallack, M. \& Kogan, N. (1965). Modes of thinking in young children. Nova Iorque: Holt, Rinehart and Winston.

Wechsler, S. M. (1999). Avaliação da criatividade: um enfoque multidimensional. Em S.M. Wechsler \& R.S.L. Guzzo (Orgs.), Avaliação psicológica: uma perspectiva internacional (pp.231-260). São Paulo: Casa do Psicólogo.

Wechsler, S. M. (2008). Criatividade: descobrindo e encorajando. São Paulo: Psy.

Woolfolk, A. E. (2000). Psicologia da educação. Porto Alegre: Artes Médicas.
Recebido em 01/09/2012

Reformulado em 05/03/2013

Aprovado em 14/05/2013 
Nota das autoras:

As autoras agradecem ao CNPq pela concessão de bolsa de Iniciação Científica à segunda autora.

Sobre as autoras:

Tatiana de Cássia Nakano é doutora em Psicologia, docente do curso de pós-graduação stricto sensu em Psicologia da PUC-Campinas e pesquisadora na linha de Instrumentos e Processos em Avaliação Psicológica. Atua principalmente na temática da criatividade e altas habilidades.

Lívia Rech de Castro é psicóloga formada pela PUC-Campinas.

Contato com as autoras:

Departamento de Pós-Graduação em Psicologia da PUC-Campinas Avenida John Boyd Dunlop, s/n. - Jardim Ipaussurama - CEP 13060-904 - Campinas-SP, Barsil. E-mail: tatiananakano@hotmail.com 